\title{
A Survey on VLC Based Massive MIMO-OFDM for 5G Networks
}

\author{
Keerti Gupta1, Neetu Sikarwar² \\ 1Student, ${ }^{2}$ Professor \\ Institute of Engineering Jiwaji University, Gwalior, Madhya Pradesh, India
}

\begin{abstract}
Exponential growth of number of mobile users has led to the increasing demand for bandwidth and channel capacity. Thus 5G has to support new multitude of various mobile applications. The $5 \mathrm{G}$ networks not only has capacity constraints but also existing challenges such as network reliability, coverage, energy efficiency and latency with current communication systems. Visible light communication provides broadband wireless data connectivity for high capacity channels. VLC system utilizes intensity modulation, where the information is conveyed through the intensity of LEDs and detected by photodiodes (PDs) at the receivers. In this survey paper the various techniques for supporting $5 \mathrm{G}$ networks using massive MIMO - OFDM concept using visible light technology have been discussed.
\end{abstract}

\section{KEYWORDS: MIMO (Multiple Input Multiple Output), Orthogonal Frequency Division Multiplexing, VLC, Spatial Multiplexing}

\section{INTRODUCTION}

This Massive MIMO has been widely recognized as a key enabling technology for future $5 \mathrm{G}$ communications due to its high system capacity and energy efficiency [2]. Massive MIMO can be realized by millimetre wave frequency ranging few GHz. In this survey, massive MIMO is combined with OFDM (Orthogonal Frequency Division Multiplexing) which is one of the viable schemes in achieving higher data rates in VLC because of high spectral efficiency, high signal-to-noise ratio(SNR)[12]. OFDM is a multiple sub-carrier system which reduces symbol rate and power consumption by serial transmission of symbols using single carrier in wide frequency range. It increases bandwidth and capacity of the channel by receiving parallel symbols in narrow frequency range, ultimately increasing the channel speed. The function of the MIMO technology mainly depends on precoding, spatial multiplexing and diversity coding techniques. Beam forming is also one of the key technologies for MIMO, mainly to reduce multipath fading effect and to increase the received signal gain. In this paper, let us discuss about the various MIMO techniques that can be used for improving $5 \mathrm{G}$ cellular network architecture. This includes various parameters such as channel state information (CSI) acquisition, beam-forming technique, spatial multiplexing, pilot contamination, millimetre wave (mm-Wave) RF modelling, DSP co-design, cognitive radio networks, cloud computing and small cell networks(SCN). From a high level perspective evolution of $5 \mathrm{G}$ provides an integrated approach

\section{VARIOUS MIMO TECHNIQUES}

This paper provides an insight of integrated technologies of MIMO technology combined with other feasible techniques for implementing $5 \mathrm{G}$ networks.

\section{A. Massive MIMO Beam Forming:}

MIMO is the method of multiplying the capacity of a radio link using multiple transmit and receive antennas to exploit multipath propagation [1]. It can also be applied to the power - line communications. It uses multiple antennas to reflect the signal which provides high gain in channel robustness and throughput [3]. Beam forming in context of MIMO essentially implies transmission in one spatial dimension to increase the gain of the antenna. Beam forming techniques are used to create a certain required antenna directive pattern under the given conditions. In the Fig.1 [1], smart antennas are used which can be controlled automatically the required pattern.

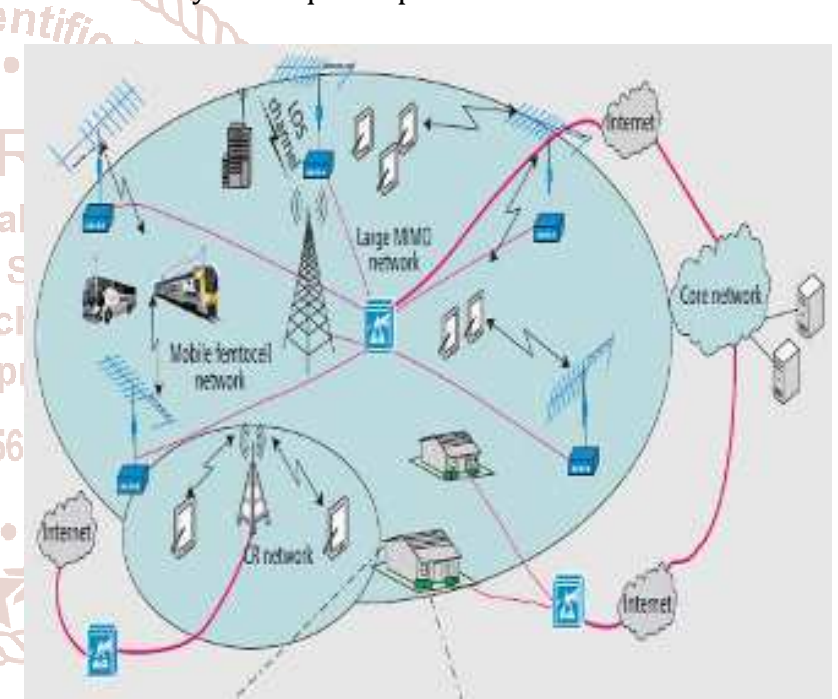

Fig.1. Heterogeneous Beam Forming Network

Massive MIMO using large antenna arrays leverage and extend benefits of 4G MIMO systems. Combining with beam forming to provide high spatial gain, especially needed for $5 \mathrm{G}$ systems that use millimetre waves.

\section{B. Massive MIMO OFDM:}

Orthogonal Frequency Division Multiplexing (OFDM) is a multiple carrier system. It can decrease the symbol rate there by reducing the power consumption of the system. It can also able to increase the bandwidth and capacity of the channel which ultimately increases the speed of the system. In current wi-max technology uses around 2000 carriers for transmitting the data through the channel. But in OFDM subsystems it can uses more number of subcarriers to increase the channel capacity. In OFDM the subcarriers are orthogonal to each other, hence cross talks are avoided. So the design of transmitter and receiver antenna system is easy compared to the traditional antenna techniques. 


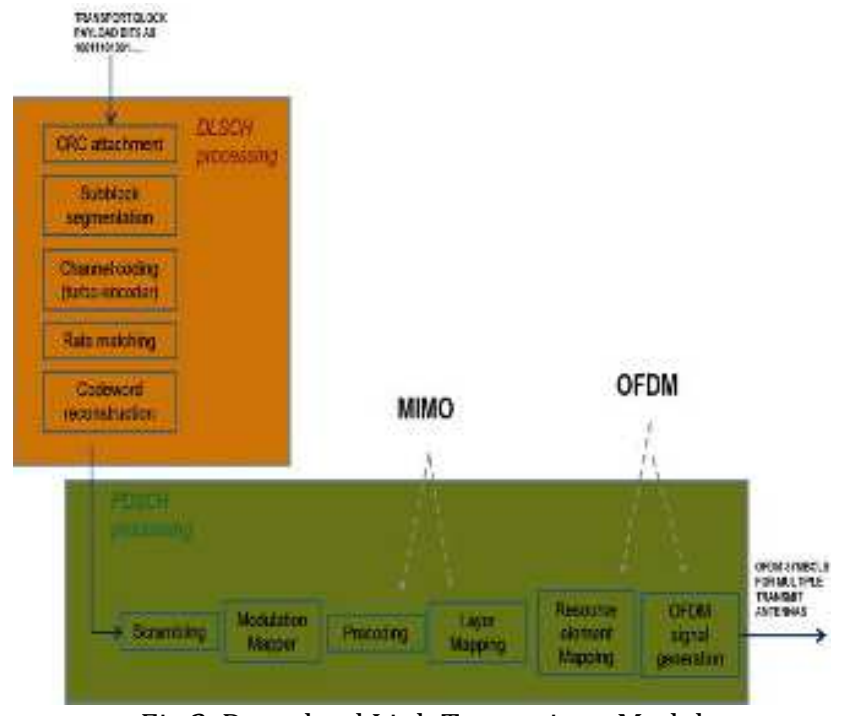

Fig.2. Download Link Transmitter Model

In the Fig. 2 concept of massive MIMO OFDM transmitter model has been demonstrated [8]. The main segment of MIMO contains precoding and layer mapping depending on the number of antennas has been used.

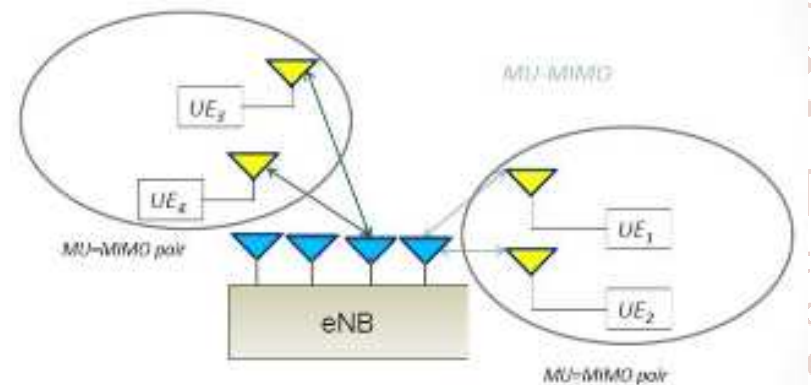

Fig.3. Massive MIMO Model

In the Fig.3 massive MIMO model used for OFDM operations has demonstrated with four user terminals which can be communicated through the diversity antennas using OFDM technique [6].

OFDM extends directly to MIMO channels with the IFFT/FFT and CP operations being performed at each of the transmit and receive antennas [7]. MIMO-OFDM decouples the frequency-selective MIMO channel into a set of parallel MIMO channels with the input-output relation for the $\mathrm{i}^{\text {th }}(\mathrm{i}=$ $0,2 \ldots L-1$ ) tone,

$$
Y_{i}=H_{i} S^{i}+N_{i} \text { where } i=0,2 \ldots L-1
$$

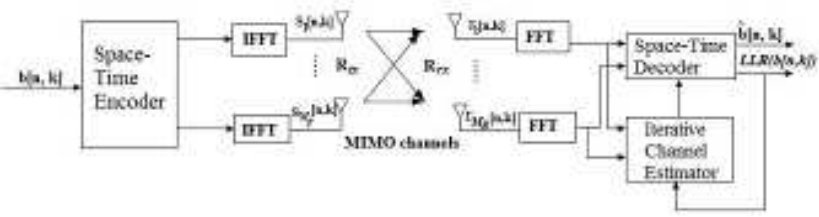

Fig.4. Broadband MIMO-OFDM Model

In the Fig. 4. An overview of broadband MIMO-OFDM model for channel estimation is shown [8]. The input data stream is encoded by a space-time or space-frequency encoder. Then, the coded data is divided into small streams of data using IFFT method and further transmitted using MIMO transmit antenna using precoding and layer mapping techniques. Each data in the transmitter antenna uses OFDM technique for modulation and further transmitted. Similarly at the receiver side the modulated data is received and demodulated using OFDM demodulator and FFT is done with the following data stream and further processed using spacetime decoder and channel estimator. Various algorithms can be used to recover the data using channel estimator and finally desired data is obtained [9].

The different channel coding techniques that can be adapted by MIMO-OFDM includes convolution coding, Reed Solomon coding, turbo coding etc. In convolution coding techniques includes various coding blocks which mainly consist of a group of shift registers and a parallel block of modulo-2 adder and the coder output. Reed Solomon code is a cyclic block codes where the polynomial function $f(x)$ is used which is the highest polynomial factor and which cannot be further integrated into lowest power orders .In turbo coding technique an inter-leaver block is used between two block codes and further processed using Viterbi Algorithm for processing the OFDM data models [8].

In OFDM, the subcarriers are orthogonal to each other and hence cross talks are avoided. It combines multi-antenna technology with the multi-carrier system to enable higher bit rate and high capacity data transmission. MIMO-OFDM system encompasses strategies like spatial multiplexing, beam forming, transmit diversity and pilot contamination. The transmit channel is encountered with various multipath fading such as large scale fading, small scale fading, interference and noise. In order to overcome all these channel effects various coding techniques can be implemented. Various mathematical MIMO channel models have been proposed for multipath propagation. OFDM is the best method suitable for multipath propagation model for massive MIMO concept [11].

\section{Massive MIMO VLC:}

Visible Light Communication provides a potential access for $5 \mathrm{G}$ wireless systems. It is an energy efficient system which uses ultra wide bandwidth there by increasing the data rate of communications [4]. It supports point-to-point LOS communications but researches been carried out for NLOS (Non Line-Of-Sight) using the scattering phenomenon and converging the scattered signal by the antenna and propagating to the receiver.

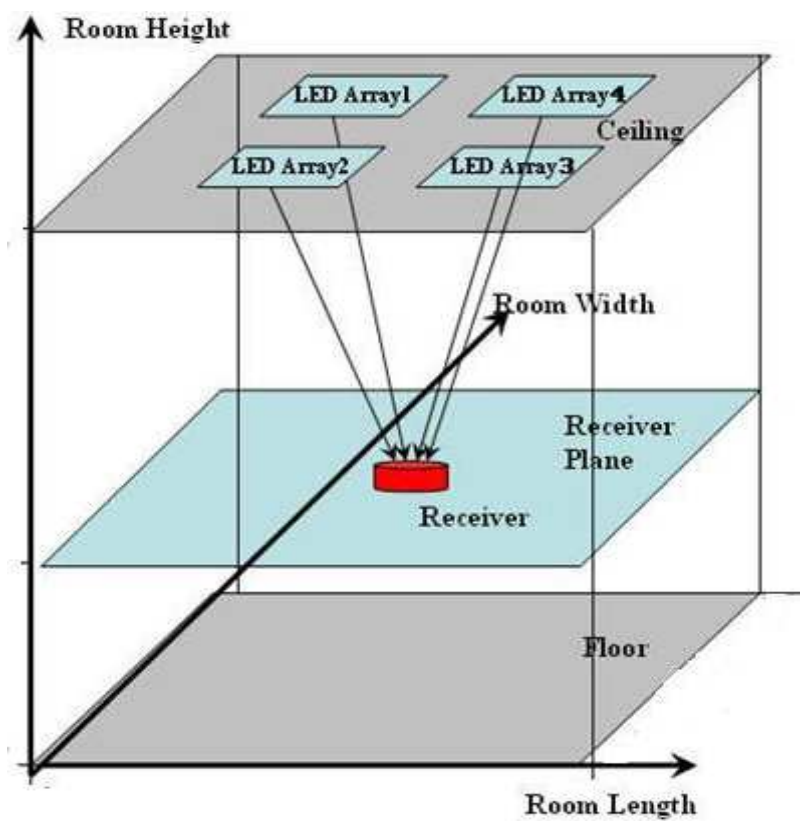

Fig.5. A Massive MIMO VLC System 
An MU-MIMO VLC system is illustrated in Fig. 5, where a single room is equipped with multiple LED units for illumination, which transmits information to multiple users simultaneously [5]. VLC offers high SNR and visibly secure channel. In current technology data communication at higher $\mathrm{GHz}$ is highly challenging. In order to communicate in this unlicensed band with increased channel capacity massive MIMO is used with large array of antennas at base transreceiver stations. Here OFDM modulation is used combined with VLC to reduce multi-user interference and to improve BER (bit error rate). Considering the distances of the multiple transmitter-receiver links are different, their temporal delays are also different, resulting in complex channel gain and phase differences when transformed to the frequency domain. The phase difference cannot be neglected when wide-band systems are considered, especially for the subcarriers with high frequencies. The precoding matrix is calculated for each subcarrier in OFDM to eliminate multiuser interference. Real channel matrices can be used for precoding, which reduces the channel correlation with one more degree of freedom and improves the system performance [5].

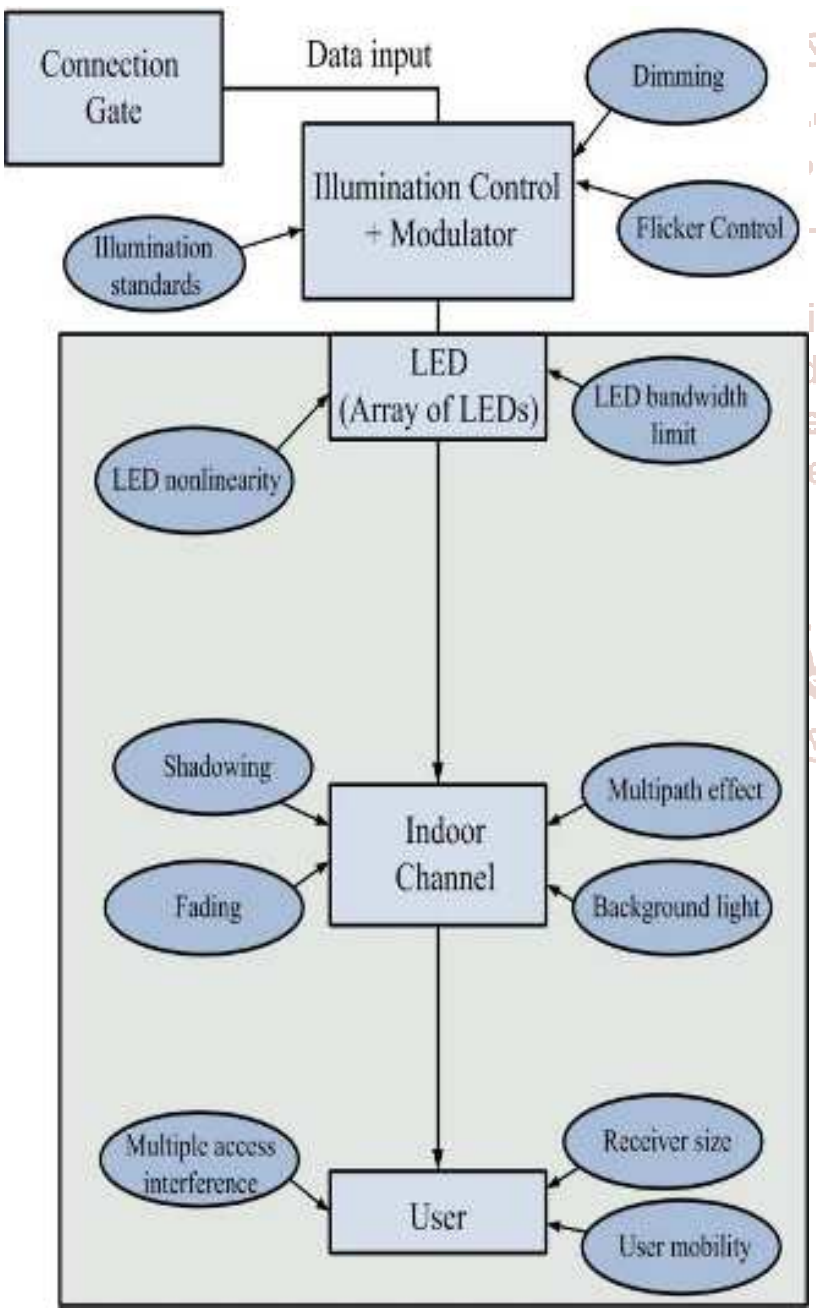

Fig.6. Challenges of VLC communications

In the Fig. 5 provides all the challenges for designing a VLC system in the real time communication media [10].

\section{FUTURE CHALLENGES IN MIMO TECHNOLOGY}

The future $5 \mathrm{G}$ networks require large bandwidth occupying large number of users for very high speed data communications. Equipping cellular base station with large number of antennas relatively provides inter symbol interference thereby causing fading effects in the path of propagation. Combining VLC with Massive MIMO is another big challenge due to the channel constraint and line of sight propagation. Signal processing challenges and channel estimation of MIMO also plays a vital role in designing of the system and implementing in the real time.

\section{CONCLUSION}

In this survey paper a detailed analysis of massive MIMO techniques had been discussed. It also provides information about various MIMO techniques combined with the beam forming, OFDM and visible light communication. Implementing VLC based MIMO - OFDM provides an ongoing research in the $5 \mathrm{G}$ technology which provides maximum constraints in implementing the scheme. This paper provides an overall view for implementing the methodology. The ongoing research includes combining the massive MIMO with OFDM and communicating using VLC technique in the high frequency unlicensed band to provide high data rate and high capacity for the $5 \mathrm{G}$ networks. Various algorithms have to be implemented for supporting this future technological need.

\section{REFERENCES}

[1] C.X. Wang, F. Haider, X. Gao, X. You, D. Yuan, H.M. Aggoune, H. Hass, "Cellular Architecture and Key technologies for $5 \mathrm{G}$ wireless communication networks" IEEE Communication Magazine, vol. 52, issue: 2, pg. 122130, February 2014.

[2] Gao, Zhen, Linglong Dai, Wei Dai and Z Wang, "Block compressive channel estimation and feedback for FDD massive MIMO", IEEE conference on Computer Communications Workshops (INFOCOM WKSHPS), 2015.

[3] E.G Larson, O.Edfors, F. Tutresson, T.L. Marzetta, "Massive MIMO for Next Generation Wireless Systems" IEEE Communication Magazine, vol. 52, issue: 2, ISSN: 0163-68044, February 2014.

[4] D. Tsonev, S. Videv, Harald Haas, "Towards a $100 \mathrm{~Gb} / \mathrm{s}$ Visible Light Wireless Access Networks", Optical Society of America (OSA), vol. 23, no.2, January 2015.

[5] Qi Wang, Zhaocheng Wang, Linglong Dai, "Multi-user MMO-OFDM for Visible Light Communications", IEEE Photonic Journal, vol.7, no.6, December 2015.

[6] Jiang Jinga, Wang Ni, "A Low Complexity Channel Estimation Algorithm for Massive MMO System", International Journal of Grid Distribution Computing, vol.7, no.4, 2014.

[7] Hanna Marshoud, Diana Dawoud, V M Kapinas, G K Karagiannidis, "MU-MIMO Precoding for VLC with imperfect CSI", $4^{\text {th }}$ International Workshop on Optical Wireless Communication (IWOW), IEEE Conference publications, pg. 93-97, 2015.

[8] Abhishek Tripathi, Komal Arora, "Different Channel Coding Techniques in MMO-OFDM", IOSR Journal of Electronics and Communication Engineering, vol.9, issue:5, pg. 65-68, October 2014.

[9] H. S. Shwetha, Sathish R N, "The Performance Analysis of MIMO OFDM System with different M-QAM Modulation and Convolution Channel Coding", IJERT, vol.4, issue:04, ISSN; 2278-0181, April 2015. 
[10] Zhen Zhan, Min Zhang, Dahai Han, Peng fei Luo, "1.2 Gbps Non-Imaging MIMO-OFDM scheme based VLC over Indoor Lighting LED Arrangements", Opto- Electronics and Communication Conference(OECC), IEEE, pg. 1-3, July 2015.

[11] D W Kwan Ng, Robert Schober, "Spectral Efficiency in Large-Scale MIMO-OFDM System with Per-Antenna Power Cost", ASILOMAR, IEEE Conference, pg. 289-29, 2012.
[12] Liang Wu, Zaichen Zhang, Huaping Lin, "MIMO-OFDM Visible Light Communication System with Low Complexity", IEEEConferenceon Communications, pg. 3933-3937, ISSN: 1550-3607, June 2013.

[13] A H Azhar, T Tran, D O’Brien, “A Giga bit/s Indoor Wireless Transmission using MIMO-OFDM Visible Light Communication", IEEE Photonics Technology Letters, vol.25, issue: 2, ISSN: 1041-1135, January 2013. 